and hepcidin. Frataxin levels in LVW were extremely low at less than $15 \mathrm{ng} / \mathrm{g}$ wet weight (normal: $214.1 \pm 81.2$ ). On crosssections, cardiomyocytes were significantly larger than normal with case means ranging from $635-1856 \mu \mathrm{m}^{2}$ for LVW and 483-1150 $\mu^{2}$ for VS (normal LVW, 140-460; normal VS, 237-613). Fe accumulations varied from minute granules to coarse aggregates in fibers undergoing phagocytosis. Measured by XRF, regional $\mathrm{Fe}$ concentration in LVW and VS were significantly increased while $\mathrm{Zn}$ remained normal. Total heart $\mathrm{Fe}$ and $\mathrm{Zn}$ did not differ from normal levels. Cytosolic and mitochondrial ferritins exhibited extensive co-localization, representing translational and transcriptional responses to $\mathrm{Fe}$, respectively. All cases met the criteria of myocarditis. Inflammatory cells contained CD68 and ferritin, and most expressed the Fe-regulatory hormone hepcidin. In conclusion, inflammation plays a major role in the pathogenesis of FA cardiomyopathy, and hepcidin-induced retention of $\mathrm{Fe}$ in macrophages contributes to cardiac damage in FRDA.

\section{Maturation of the Fetal Olfactory Bulb}

\section{Harvey B. Sarnat}

University of Calgary, Calgary, Alberta, Canada

doi:10.1017/cjn.2015.256

The olfactory bulb exhibits architecture unique amongst laminar cortices, lacking molecular and subplate zones and having superficial synaptic glomeruli. Its ontogenesis also is unique because neuroblasts do not migrate radially but stream in from the rostral telencephalon; an ependymal-lined olfactory ventricle is transitory. The olfactory is the only sensory system to not project to the thalamus but incorporates a thalamic equivalent. It is a repository of progenitor cells in the mature brain. The aim was to define olfactory bulb development in the human foetus: synaptogenesis and cellular maturation.

Immunoreactivity in paraffin sections of synaptophysin, NeuN, calretinin, vimentin and nestin was examined at autopsy in olfactory bulb in 20 foetuses, 9-40wks gestation. Synaptophysin reactivity was seen around the somata of mitral and tufted neurons at 9 wks, synaptic glomeruli at 13 wks. The granule cell layer in the core exhibited NeuN-reactive nuclei in cells of the outer half at 20wks; $60 \%$ of granular neurons reacted by term. Synaptophysin reactivity in the granular layer initiates at $15 \mathrm{wk}$. GABAergic calretinin-reactive neurons and neurites and synaptic glomeruli appeared at $13 \mathrm{wks}$. Nestin- and vimentin-reactive bipolar progenitor cells were shown at all gestational ages, mainly in the granular layer, the ratio to other cells remaining constant. Synapses form in the small accessory olfactory bulb of the nervus terminalis earlier than in the main bulb. Development of synaptic vesicles in the human fetal olfactory bulb is precise both spatially and temporally, but not yet fully mature at term.

In brain malformations and congenital metabolic and genetic diseases, the olfactory bulb may be affected and provide additional neuropathological data. Therapeutic autologous transplantation of olfactory progenitor cells focus renewed interest in the olfactory bulb.

\section{Congenital Lymphocytic Choriomeningitis Virus: A Neuropathological Study}

\section{Fallet-Bianco}

CHU Sainte-Justine, Montréal, Québec, Canada

doi:10.1017/cjn.2015.257

Lymphocytic choriomeningitis virus (LCMV) carried and secreted by mice, infects great numbers of people. LCMV infection acquired during childhood or adulthood is usually moderately symptomatic with a full recovery. When the infection occurs prenatally, it results in a wide spectrum of severe brain lesions described mainly on imaging. Neuropathological data have never been reported.

We present 2 fetuses with a prenatal diagnosis of microcephaly with ventriculomegaly, abnormal gyration, and ponto-cerebellar hypoplasia in one case. Parents elected to terminate the pregnancy. A complete autopsy demonstrated no dysmorphic features, no visceral or skeletal malformation. Histological examination of viscera did not show any significant lesion.

Neuropathological examination confirmed microcephaly and ventriculomegaly with a thick yellowish band surrounding the ventricles. Identical histological lesions were observed in both cases associating a polymicrogyria and a diffuse necrosis of parenchyma with massive calcifications all around the ventricles. The most characteristic feature was the unusual aspect of necrosis, distinct from that observed in other infections, characterized by a finely granular appearance looking like sand. Small lymphocytic infiltrates were observed in the leptomeninges and in the choroid but not in the retina. The congenital LCMV infection was confirmed by serologic testing.

This study confirms the strong neurotropism of LCMV and demonstrates that prenatal infection has some particular features such as absent systemic signs, and distinct appearance of the necrosis that allow to distinguish it from other congenital infections and other non infectious conditions.

\section{Cerebral hyaline astrocytic inclusions in treatment- resistant epilepsy and global developmental delay} \section{Signaevski ${ }^{1}$, C. Sanguansermsri ${ }^{1}$, M. Connolly ${ }^{1}$, LN. Hazrati ${ }^{2}$,} C. Dunham ${ }^{1}$

${ }^{1}$ University of British Columbia, Vancouver, Canada; ${ }^{2}$ University of Toronto, Toronto, Canada

doi:10.1017/cjn.2015.258

Cerebral hyaline astrocytic inclusions (HAI) have been observed in a subset of patients with epilepsy, structural brain anomalies, and developmental delay. We present a case of a 2.5-year-old male with epilepsy and global developmental delay. Chromosomal microarray detected a copy loss at 22q13 that resulted in a partial deletion of SHANK3 gene. The EEGs revealed seizure activity arising from left frontal central region. Invasive video electrocorticography captured clusters of epileptic spasms, all originating from left antero-lateral frontal lobe rostral to the motor cortex. We utilized routine histology to identify the inclusions and mapped their distribution in the resected portion of 